\title{
Rivalidade ou Violência? A percepção de Estudantes Universitários Vítimas de Violência por parte de Irmãos
}

\author{
Inês Carvalho Relva ${ }^{1}$ \\ Universidade de Trás-Os-Montes e Alto Douro \\ Centro de Estudos Sociais da Universidade de Coimbra \\ Madalena Alarcão \\ Centro de Estudos Sociais da Universidade de Coimbra \\ Otília Monteiro Fernandes \\ Universidade de Trás-Os-Montes e Alto Douro
}

\begin{abstract}
RESUMO - Este estudo pretende avaliar a percepção das vítimas relativamente a comportamentos de cariz físico, sexual e psicológico, perpetrados por irmãos. Faz parte de uma investigação mais vasta na qual foram avaliados os diferentes tipos de violência e a sua frequência no início da adolescência. Neste estudo, os participantes classificaram os abusos sofridos com recurso ao Self-Labelling of Personally Experienced Violence. Os resultados indicam que os jovens que sofreram todos os tipos de vitimizações (físicas, psicológicas e sexuais) atribuíram os incidentes à "rivalidade entre irmãos", de modo significativo, quando comparados com os jovens que só sofreram um ou dois tipos de vitimização (psicológicas, físicas, psicológicas + físicas ou psicológicas + sexuais). Os resultados são discutidos segundo perspectivas de 'normalização de agressões', as quais explicam a manutenção de comportamentos abusivos contra irmãos.
\end{abstract}

Palavras-chave: rivalidade, violência, irmãos, percepção

\section{Rivalry or Violence? Perception of University Students Victims of Sibling Violence}

\begin{abstract}
This study aimed to investigate the perception of victims of sibling violence (physical, sexual and psychological). It is part of a larger investigation that analyzed the different types of violence and its frequency in early adolescence. In the present study, the participants classified the mistreatments they suffered on the Self-Labelling of Personally Experienced Violence. In the explanation of the occurrences, youngsters enduring all types of sibling victimization (physical, sexual and psychological) attributed "sibling rivalry" significantly more importance than their colleagues who were victims of only one or two violence type(s) (physical, psychological, or psychological + physical + or psychological + sexual). The results are discussed according to 'normalization of aggression' perspectives that help to understand the maintenance of abusive behavior towards siblings.
\end{abstract}

Keywords: rivalry, violence, siblings, perception

Foi só nos finais da década de 1970 que surgiram os primeiros estudos sistemáticos que tentam caracterizar e compreender o fenómeno da violência entre irmãos. Desses destacam-se: a National Family Violence Survey (Straus, Gelles, \& Steinmetz, 1980) e a única sondagem que avaliou, retrospetivamente, as interações sexuais entre irmãos (Finkelhor, 1980).

Diversos estudos confirmaram frequências elevadas de comportamentos violentos entre irmãos, particularmente durante a infância (Graham-Bermann, Cutler, Litzenberger, \& Schwartz, 1994; Hardy, 2001; Hardy, Beers, Burgess, \& Taylor, 2010; Straus et al., 1980), e apesar de parecerem decrescer com a entrada na adolescência (Noland, Liller, McDermott, Coutler, \& Seraphine, 2004), a gravidade das vitimizações parece ser independente da idade (Finkelhor, Turner, \& Ormond, 2006) e estes comportamentos abusivos

1 Endereço para correspondência: Universidade de Trás-os-Montes e Alto Douro, Escola de Ciências Humanas e Sociais, Departamento de Educação e Psicologia, Quinta de Prados, Vila Real, Portugal, 5000801.E-mail:irelva@utad.pt parecem iniciar-se desde muito cedo. Turner, Finkelhor, Ormrod e Hamby (2010) reuniram dados de 503 crianças com idades até aos 2 anos, a partir dos seus cuidadores, e verificaram uma taxa anual de vitimização de $15,4 \%$ às mãos de irmãos mais velhos ou de idades aproximadas.

Embora escassos, os dados disponíveis relativos à violência extrema entre irmãos são preocupantes. Num estudo junto de crianças $(n=323)$ provenientes de contextos clínicos e não-clínicos verificou-se uma taxa de vitimização por parte dos irmãos de aproximadamente $80 \%$ (Kolko, Kazdin, \& Day, 1996). Nos anos mais recentes, também Kettrey e Emery (2006) reportaram percentagens superiores a 70\% de vitimização grave perpetrada por irmãos. Na sociedade norteamericana, esses dados podem contribuir para explicar as incidências anuais, entre 7,6\% (Underwood \& Patch, 1999) e 10\% (Dawson \& Langan, 1994), de homicídios ocorridos em contexto familiar que são perpetrados por irmãos da vítima.

No que concerne às interações sexuais entre irmãos, a única sondagem comunitária de que temos conhecimento e que avaliou este problema, numa amostra de 796 estudantes 
universitários, revelou que $15 \%$ das moças e $10 \%$ dos rapazes haviam interagido sexualmente com irmãos e que, destes, 25\% tinham sido coagidos a tal (Finkelhor, 1980). Já Wiehe (1997) recrutou 150 adultos através de anúncios e obteve uma frequência de $67 \%$ de vítimas de abuso sexual perpetrado por irmãos, predominantemente durante a infância. Claramente, os aspectos metodológicos de amostragem e recolha de dados justificam a variabilidade destas taxas. Anos mais tarde, Caffaro e Conn-Caffaro (1998) estudaram 73 adultos vitimizados por irmãos e verificaram que 39\% dos participantes tinham sofrido abusos sexuais recorrentemente. Se considerado a partir de processos judiciais de vitimização sexual, o número de crianças que são forçadas por um irmão a ter relações sexuais oscila entre os 3\% (Alpert, 1997) e os 13\% (Finkelhor, 1980), frequências compreensivelmente baixas devido à relutância de pais e vítimas em oficializar uma denúncia.

Contudo, e apesar da elevada prevalência da violência entre os irmãos, os discursos em torno desta forma de violência (que pode assumir diferentes tipos: física, psicológica e/ou sexual) têm sido ignorados por grande parte da comunidade científica, mas também pela comunidade em geral (Phillips, Phillips, Grupp, \&Trigg, 2009). Continua a presumir-se que quando as crianças ou adolescentes batem nos pares ou irmãos não é tão sério como quando os mesmos atos são perpetrados por adultos (Finkelhor et al., 2006), apesar de diversos estudos sugerirem que a violência entre irmãos é a forma mais prevalente de violência na família (ex., Eriksen \& Jensen, 2009; Wiehe, 1997) e que pode, até, ter consequências fatais (Kiselica \& Morrill-Richards, 2007).

São vários os constrangimentos ao estudo sistemático deste fenómeno. Primeiro, as crenças de que a violência entre irmãos constitui uma experiência normativa do desenvolvimento ou uma oportunidade de aprendizagem de resolução de conflitos (Finkelhor et al., 2006) têm ainda grande aceitação cultural e perpetuam a "reinterpretação" do comportamento violento entre irmãos como mera manifestação de rivalidade (Kettrey \& Emery, 2006). Pais, educadores, agentes sociais, mas também, surpreendentemente, as próprias vítimas (Hardy, 2001), adequam as suas atribuições minimizando o impacto dessas experiências (Hardy et al., 2010). Consequentemente, é rara a criminalização da violência quando esta ocorre entre irmãos (Kiselica \& Morrill-Richards, 2007; Krienert \& Walsh, 2011a).

Desta forma, os episódios de violência entre irmãos são subdeclarados, o que dificulta a obtenção de uma casuística populacional. Este facto acarreta várias consequências perversas. Por um lado, resulta num número limitado de estudos que examinam o fenómeno na comunidade, tornando quase impraticável o estudo da incidência do fenómeno (Finkelhor et al., 2006; Krienert \& Walsh, 2011a). Consequentemente, pela não detecção de casos, perde-se a oportunidade de intervenção direta com essas crianças (vítimas e perpetradores, e, eventualmente, testemunhas desses atos violentos) e, também, indireta, noutros tipos de violência perpetrados pelos abusadores (ex., bullying aos colegas). Por outro lado, a escassez de estudos sistemáticos contribui para o não reconhecimento social do problema (Eriksen \& Jensen, 2009).
Finalmente, algumas limitações metodológicas enfraquecem os estudos disponíveis sobre a violência entre irmãos. Questões de poder amostral decorrentes de amostras clínicas pequenas e da ausência de grupos de comparação (ex., Smith \& Israel, 1987), défices na representatividade amostral das várias faixas etárias (ex., Daie, Witztum, \& Eleff, 1989), métodos de recolha de dados suscetíveis de viés (ex., sondagens telefónicas tomando exclusivamente os pais das crianças como informantes; Finkelhor et al., 2006), entre outras, estarão seguramente implicadas na discrepância das frequências anteriormente referidas. Se a estas se juntarem questões conceptuais de base, como o uso de definições inconsistentes das várias formas de violência entre irmãos (Krienert \& Walsh, 2011a; Krienert \& Walsh, 2011b), compreende-se que este fenómeno tenha "contornos ambíguos", que seja difícil o controlo estatístico dos vários fatores causais envolvidos (Stock, 1993), e que, com algumas exceções (ex., Hardy, 2001; Krienert \& Walsh, 2011a; Krienert \& Walsh, 2011b), ocorra um hiato na literatura quanto à caracterização de vítimas, perpetradores e incidentes de violência. Alguns autores (ex., Eriksen \& Jensen, 2009; Kettrey \& Emery, 2006) têm levantado esta questão da ambiguidade conceitual que rodeia a investigação no âmbito da violência entre irmãos. A revisão da literatura permite confirmar que termos como abuso, violência, agressão, conflito, rivalidade e bullying têm sido utilizados, de forma pouco diferenciada, para se referirem aos mesmos comportamentos (Relva, Fernandes, \& Alarcão, 2012).

Num estudo anterior (Relva, Fernandes, Alarcão, \& Martins, 2014) verificou-se que a frequência da violência entre os irmãos, numa amostra comunitária de estudantes universitários portugueses, é muito elevada no início da adolescência. Os participantes admitiram ter perpetrado atos de violência psicológica, física e sexual (88.4\%, 69.2\% e $6.3 \%$ respectivamente) para com os irmãos, durante o período de referência considerado (13 anos de idade). Para o mesmo período, as taxas de vitimização psicológica, física e sexual por irmãos foram, respectivamente, $87.2 \%$, $69.2 \%$ e $7.5 \%$. Para ambas as formas de violência (física e psicológica) os resultados aproximam-se dos valores indicados por diversos estudos internacionais (ex., GrahamBermann et al., 1994), sugerindo que se trata de um fenómeno transcultural (Relva et al., 2012). No que concerne à violência sexual, as percentagens do abuso sexual reportadas foram semelhantes às verificadas noutros estudos (Finkelhor, 1980; Hardy, 2001). Deste modo, o presente estudo, partindo das frequências de vitimização acima referidas, pretende avaliar a percepção das vítimas quanto aos comportamentos de natureza psicológica, física e/ou sexual de que foram alvo.

\section{Método}

\section{Participantes}

Quinhentos e oitenta e oito estudantes universitários (219 do sexo masculino), com idade média de 20 anos $( \pm 4)$, todos com irmãos, consentiram em participar no estudo. Cerca 
de $65 \%$ da amostra possuía apenas um irmão, 24\% tinha dois, $7 \%$ três, e os restantes $4 \%$ tinham quatro ou mais. Foi solicitado aos participantes que indicassem o sexo do irmão com quem experimentaram mais conflitos durante o período de referência etária - 13 anos. De igual modo, solicitou-se que indicassem a idade que o irmão tinha nesse período. Com base nestas informações, determinou-se a composição das díades fraternas e a posição do participante na fratria. As principais variáveis sociodemográficas estão descritas na Tabela 1.

Tabela 1. Características Sociodemográficas dos Participantes

\begin{tabular}{|c|c|}
\hline Variável & n (\%) \\
\hline \multicolumn{2}{|l|}{ Sexo } \\
\hline Masculino & $219(37.2 \%)$ \\
\hline Feminino & $369(62.8 \%)$ \\
\hline Idade, média (DP) & $20.3(4.4)$ \\
\hline \multicolumn{2}{|l|}{ Número de irmãos } \\
\hline Um & $383(65.1 \%)$ \\
\hline Dois & $139(23.6 \%)$ \\
\hline Três & $43(7.3 \%)$ \\
\hline Quatro ou mais & $23(4.0 \%)$ \\
\hline \multicolumn{2}{|l|}{ Posição na fratria } \\
\hline Mais velho que o irmão & $285(48.5 \%)$ \\
\hline Mais novo que o irmão & $293(49.8 \%)$ \\
\hline Gémeos & $10(1.7 \%)$ \\
\hline Diferença de idade entre irmãos, média (DP) & $5.5(3.2)$ \\
\hline \multicolumn{2}{|l|}{ Tipo de díade fraterna } \\
\hline Ambos rapazes & $95(16.2 \%)$ \\
\hline Ambas raparigas & $147(25.0 \%)$ \\
\hline Mista & $346(58.8 \%)$ \\
\hline \multicolumn{2}{|l|}{ Estado civil dos pais* } \\
\hline Casados / em união & $515(87.6 \%)$ \\
\hline Divorciados / separados & $45(7.7 \%)$ \\
\hline \multicolumn{2}{|l|}{ Escolaridade do pai* } \\
\hline Sem escolaridade & $2(0.3 \%)$ \\
\hline Primeiro ciclo & $210(35.7 \%)$ \\
\hline Segundo ciclo & $102(17.3 \%)$ \\
\hline Terceiro ciclo & $81(13.8 \%)$ \\
\hline Ensino secundário & $76(12.9 \%)$ \\
\hline Ensino superior & $55(9.4 \%)$ \\
\hline \multicolumn{2}{|l|}{ Escolaridade da mãe* } \\
\hline Sem escolaridade & $4(0.7 \%)$ \\
\hline Primeiro ciclo & $169(28.7 \%)$ \\
\hline Segundo ciclo & $100(17.0 \%)$ \\
\hline Terceiro ciclo & $101(17.2 \%)$ \\
\hline Ensino secundário & $90(15.3 \%)$ \\
\hline Ensino superior & $70(11.9 \%)$ \\
\hline
\end{tabular}

Nota. * Esta variável denota não-respostas; $D P=$ Desvio-padrão. $N=588$.

\section{Instrumentos}

As escalas Revised Conflict Tactics Scales - Sibling Version (CTS2-SP; Straus, Hamby, Boney-McCoy, \& Sugarman, 1996) foram utilizadas para estimar a frequência dos vários tipos de violência entre irmãos. Trata-se de um conjunto de cinco escalas, com um total de 39 itens, já aferidas para a população portuguesa (Relva, Fernandes, \& Costa, 2013), que permite avaliar as táticas de conflito preferencialmente utilizadas no relacionamento entre irmãos. As cinco escalas são: negociação (seis itens), agressão psicológica (oito itens), agressão física sem sequelas (12 itens), coerção sexual (sete itens) e agressão física com sequelas (seis itens). Cada item é representado por duas questões, a primeira referindo um comportamento emitido pelo sujeito em relação ao irmão/irmã (perpetração), e a segunda referindo o mesmo comportamento em sentido inverso, do irmão/irmã em relação ao sujeito (vitimização). Para este estudo foram apenas utilizadas as questões relativas à vitimização.

O Questionário Sociobiográfico (QSB) é um questionário de informação sociodemográfica, adaptado do Social Environment Questionnaire (Toman, 1993), que pesquisa as características do sujeito (ex., idade, sexo, nacionalidade, doenças, hospitalizações), da sua família (ex., com quem vive, ocupação, escolaridade e situação conjugal dos pais, condições habitacionais), e do seu subsistema fraternal (ex., número de irmãos, posição na fratria, sexo, ocupação, doenças).

O Self-Labelling of Personally Experienced Violence (SLPEV; Kettrey \& Emery, 2006) é um questionário de oito itens desenvolvido a partir do original de Hamby e GrayLittle (2000), que avalia a percepção das vítimas de violência doméstica face ao carater abusivo dos comportamentos de que foram alvo. Na versão de Kettrey e Emery (2006) é pedido ao sujeito que classifique, numa escala de 1 ("Definitivamente não") a 10 ("Definitivamente sim"), até que ponto os desentendimentos ocorridos com o irmão/irmã podem ser considerados "conflito", "rivalidade", "agressão", "violência" e "abuso". O estudo original indicou um valor de Alfa de Cronbach de .65 para a escala total (Kettrey \& Emery, 2006); no presente estudo, a fiabilidade da escala total traduziu-se num Alfa de .73, sugerindo uma boa consistência interna (Nunnally \& Bernstein, 1994).

\section{Procedimentos}

Após obtenção de autorizações institucionais, os questionários, anónimos, foram autoadministrados em turmas de diferentes universidades portuguesas. O seu preenchimento foi voluntário e sem incentivos. O horário de administração foi previamente acordado com os responsáveis das turmas, normalmente antes ou depois das aulas. A primeira autora explicitou os objetivos do estudo em cada turma e realizou o briefing dos participantes após a recolha dos instrumentos. Para evitar homogeneidade amostral, os participantes foram recrutados em cursos de diferentes áreas de conhecimento. 


\section{Procedimentos de análise e de redução de dados}

Os participantes vitimizados foram classificados consoante o tipo de violência sofrida e foram estudadas as suas respostas aos itens "conflito", "rivalidade", "agressão", "violência" e "abuso" através da prova de Kruskal-Wallis. A análise de efeitos significativos realizou-se através da prova U de Mann-Whitney com ajustamento de Bonferroni para o número de comparações. Em todas as análises foi considerado um nível de significância estatística de 5\%. No tratamento estatístico foi utilizado o Statistical Package for the Social Sciences (versão 18).

\section{Resultados}

\section{Perceção das vítimas quanto ao caráter abusivo dos comportamentos}

Os participantes vitimizados $(N=522 ; 88.8 \%$ da amostra total) foram classificados a partir das CTS2-SP consoante $\mathrm{o}(\mathrm{s})$ tipo(s) de violência sofrida $(\mathrm{P}=$ violência psicológica; $\mathrm{F}=$ violência física; $\mathrm{P}+\mathrm{F}=$ violência psicológica e física; $\mathrm{P}+\mathrm{S}=$ violência psicológica e sexual; $\mathrm{P}+\mathrm{F}+\mathrm{S}=$ violência psicológica, física e sexual) o que resultou em grupos com frequências desiguais. Em função do(s) tipo(s) de violência sofrida foram analisadas as respostas dos participantes aos itens "conflito", "rivalidade", "agressão", "violência" e "abuso". Dos resultados há a destacar o grupo que sofreu todas as formas de vitimização $(\mathrm{P}+\mathrm{F}+\mathrm{S})$ que atribuiu significativamente mais importância à "rivalidade" para explicar os incidentes ocorridos com os irmãos. A Tabela 2 descreve as medianas e amplitudes das pontuações dos grupos de vítimas nos diferentes itens do SLPEV.

$\mathrm{O}$ desvio à normalidade das distribuições dos itens do $S L P E V$ e as frequências dos grupos justificaram o uso da prova de Kruskal-Wallis nas suas pontuações estandardizadas (escores Z). A Figura 1 resume a distribuição das pontuações estandardizadas dos participantes para cada item ("conflito", "rivalidade", "agressão", "violência" e "abuso"), considerando o tipo de vitimização sofrida.

Não foram encontradas diferenças significativas entre os grupos na avaliação do caráter de "conflito" $[\chi 2(4,473)$ $=7.66, \mathrm{p}=.11]$ e de "abuso" $[\chi 2(4,473)=6.12, \mathrm{p}=.19]$ dos comportamentos do irmão, sugerindo que a avaliação destas dimensões é relativamente independente do tipo de vitimização sofrida. Quanto à "rivalidade", a prova de Kruskal-Wallis sinalizou uma diferença estatisticamente significativa entre as medianas dos grupos, $\chi 2(4,473)=$ $14.54, \mathrm{p}<.01$. Indagou-se este efeito comparando os vários pares de grupos através da prova de U de Mann-Whitney. Para controlar erros do tipo I nas dez comparações a efetuar, o nível de significância adotado passou a ser de $\mathrm{p}<.005$. Especificamente, o grupo de vitimização psicológica, física e sexual (grupo $\mathrm{P}+\mathrm{F}+\mathrm{S}$ ) atribuiu significativamente mais importância (rank médio $=79.90$ ) à rivalidade na explicação dos comportamentos abusivos do que o grupo de vitimização psicológica (grupo P; rank médio = 56.69), $\mathrm{U}=972.50, \mathrm{Z}=$ $-3.71, \mathrm{p}<.001, \mathrm{r}=0.33$, o mesmo acontecendo (rank médio = 231.81) em relação ao grupo de vitimização psicológica e física (grupo $\mathrm{P}+\mathrm{F}$; rank médio = 179.68), $\mathrm{U}=4069.50$, $\mathrm{Z}=-3.09, \mathrm{p}<.005, \mathrm{r}=0.16$. Relativamente à dimensão de "agressão", foram também detetadas diferenças significativas entre os grupos, $\chi 2(4,473)=18.81, p<.01$. O seu seguimento pelo U de Mann-Whitney indicou novamente que o grupo $\mathrm{P}+\mathrm{F}+\mathrm{S}$ (rank médio $=79.86)$ se distinguia significativamente do grupo $\mathrm{P}($ rank médio $=57.97), \mathrm{U}=1055.0, \mathrm{Z}=-4.25$, $\mathrm{p}<.001, \mathrm{r}=0.38$, e era igualmente distinto (rank médio $=$ 225.0) do grupo $\mathrm{P}+\mathrm{F}$ (rank médio $=181.37), \mathrm{U}=4480.0, \mathrm{Z}=$ $-3.02, \mathrm{p}<.005, \mathrm{r}=0.16$, pelo maior reconhecimento do caráter agressivo do comportamento do irmão abusador. Finalmente, apesar dos grupos não terem diferido significativamente na avaliação do item "violência", $\chi 2(4,473)=8.44, p=.08$, a tendência da prova de Kruskal-Wallis para a significância estatística poderá resultar de uma maior valorização desta dimensão pelo grupo $\mathrm{P}+\mathrm{F}+\mathrm{S}($ rank médio $=72.89)$ quando comparado com o grupo $\mathrm{P}($ rank médio $=60.62), \mathrm{U}=1299.0$, $\mathrm{Z}=-2.705, \mathrm{p}=.007, \mathrm{r}=0.24$.

Tabela 2. Medianas e Amplitudes das Pontuações dos Grupos de Vítimas nos Itens do SLPEV

\begin{tabular}{|c|c|c|c|c|c|}
\hline Itens & $\begin{array}{c}\text { Grupo } 1 \\
(\mathrm{P}) \\
\mathrm{n}=92\end{array}$ & $\begin{array}{c}\text { Grupo } 2 \\
\text { (F) } \\
n=8\end{array}$ & $\begin{array}{c}\text { Grupo } 3 \\
(\mathrm{P}+\mathrm{F}) \\
\mathrm{n}=\mathbf{3 3 5}\end{array}$ & $\begin{array}{c}\text { Grupo } 4 \\
\begin{array}{c}(P+S) \\
n=3\end{array}\end{array}$ & $\begin{array}{c}\text { Grupo } 5 \\
(P+F+S) \\
n=35\end{array}$ \\
\hline Conflito & $2(1-10)$ & $2(1-8)$ & $3(1-10)$ & $7(1-8)$ & $5(1-10)$ \\
\hline Rivalidade & $1(1-10)$ & $1.5(1-6)$ & $1(1-10)$ & $1(1-8)$ & $2.5(1-10)$ \\
\hline Agressão & $1(1-10)$ & $1(1-2)$ & $1(1-10)$ & $1(1-10)$ & $1(1-10)$ \\
\hline Violência & $1(1-10)$ & $1(1-2)$ & $1(1-10)$ & $1(1-10)$ & $1(1-10)$ \\
\hline Abuso & $1(1-10)$ & $1(1-1)$ & $1(1-10)$ & $1(1-10)$ & $1(1-10)$ \\
\hline
\end{tabular}

Nota. As estatísticas apresentadas são medianas (mínimo-máximo). As escalas denotaram 49 não-respondentes; $\mathrm{P}=$ Violência Psicológica; $\mathrm{F}=$ Violência Física; $\mathrm{P}+\mathrm{F}=$ Violência Psicológica e Física; $\mathrm{P}+\mathrm{S}=$ Violência Psicológica e Sexual; $\mathrm{P}+\mathrm{F}+\mathrm{S}=$ Violência Psicológica, Física e Sexual. $N=522$. 


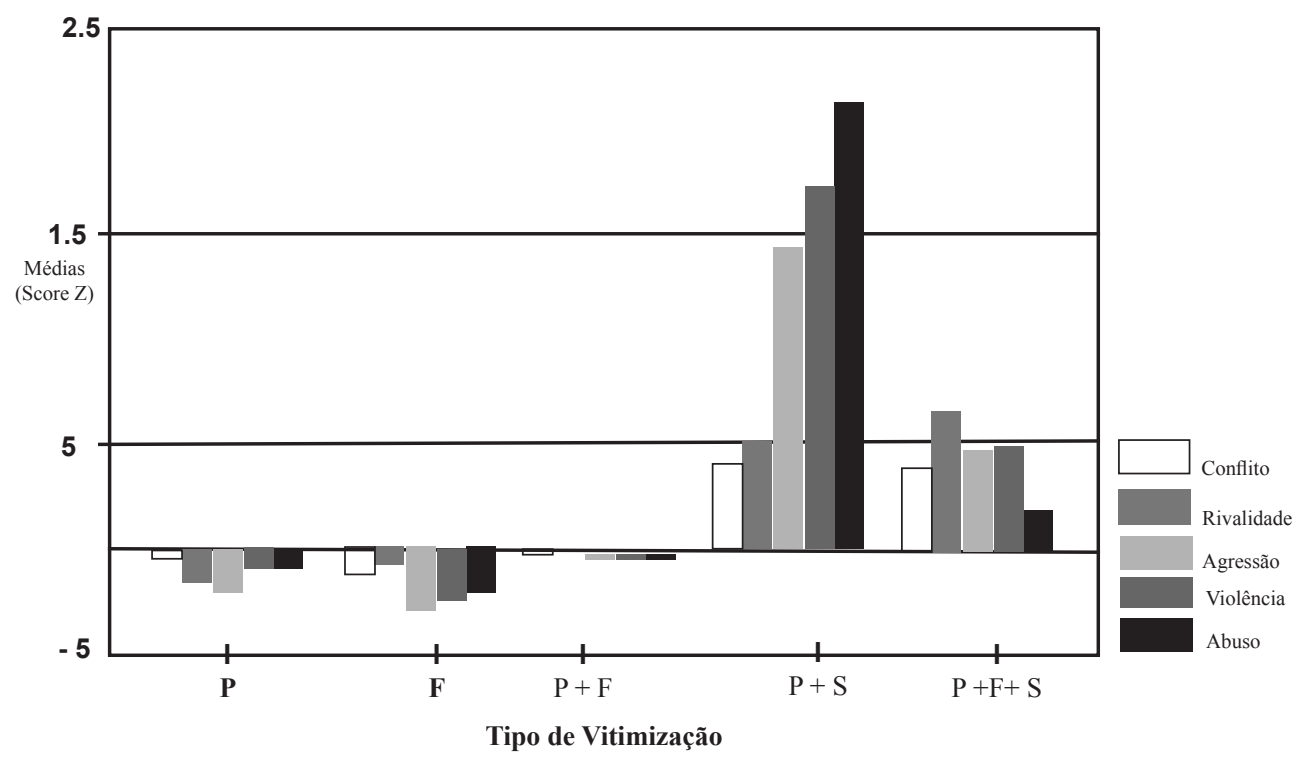

Figura 1. Pontuações Estandardizadas das Dimensões do SLPEV consoante o Tipo de Vitimização Sofrido Pelos Participantes $(N=522)$

Nota. As escalas do SLPEV denotaram perda de dados (49 não-respondentes); $\mathrm{P}=$ Violência Psicológica ( $\mathrm{n}=92$ ); $\mathrm{F}=$ Violência Física $(n=8) ; P+F=$ Violência Psicológica e Física $(n=335) ; P+S=$ Violência Psicológica e Sexual $(n=3) ; P+F+S=$ Violência Psicológica, Física e Sexual ( $\mathrm{n}=35)$.

\section{Discussão}

A violência entre irmãos é, provavelmente, a forma de violência mais comum nas famílias (ex., Straus et al., 1980) e, paradoxalmente, a mais ignorada. O presente estudo avaliou a perceção das vítimas quanto à violência reportada entre irmãos, pela administração do Self-Labelling of Personally Experienced Violence a estudantes universitários. Os resultados sugerem que alguma "dissonância cognitiva" (Hardy et al., 2010) contribui para a manutenção do fenómeno da violência entre irmãos.

A percepção dos participantes quanto ao carater das vitimizações sofridas é globalmente consistente com os resultados obtidos por Kettrey e Emery (2006) e por Hardy et al. (2010). Por um lado, as dimensões de "conflito", "abuso" e "violência" foram valorizadas de modo semelhante entre os participantes vitimizados, independentemente das formas de violência implicadas. Por outro lado, o grupo que sofreu todas as formas de vitimização $(\mathrm{P}+\mathrm{F}+\mathrm{S})$ reconheceu significativamente melhor o carater agressivo dos incidentes, mas, paradoxalmente, atribuiu significativamente mais importância à "rivalidade" entre irmãos para a sua explicação. Estes resultados corroboram as premissas das teorias de normalização da violência (Hoffman, Kiecolt, \& Edwards, 2005), que preveem o uso frequente da força física como forma culturalmente aceite de resolução de problemas. De facto, a violência entre irmãos é comummente aceite como uma experiência benigna e normativa da infância, e como uma oportunidade de serem desenvolvidas competências sociais (Finkelhor et al., 2006). Para tal crença social contribuem também as práticas educativas parentais que, ao tolerarem as interações agressivas ou sexuais entre irmãos, perpetuam nas vítimas a mensagem de que "o comportamento não é realmente abusivo" (Wiehe, 2002). Desse modo, pelo caráter dos abusos ser socialmente validado como "inócuo" e os ofensores desresponsabilizados aos olhos dos pais e agentes educativos, resta aos irmãos violentados "reinterpretarem" a realidade e a frequência dos abusos, respondendo assim às expectativas que as normas sociais lhes incutem (Kettrey \& Emery, 2006). Ao referirem-se aos incidentes como "rivalidade", os participantes mais vitimizados passam a considerá-los como normativos (Fernandes, 2002). No entender de Hardy et al. (2010), através desta normalização das experiências de vitimização, as vítimas permitem-se reduzir a dissonância cognitiva que duas atitudes de valências opostas lhes provocam: simultaneamente acreditar que os irmãos se amam e sofrer/temer ser violentado por eles. Consequentemente, não só os vários tipos de abuso deixam de ser considerados como "violência", como as próprias vítimas passam a ter dificuldade em reconhecer-se como tal, perdurando o estereótipo de que a violência entre irmãos é simplesmente uma forma "pueril" de agressão (Finkelhor et al., 2006). Estudos futuros e programas de prevenção/ intervenção que sejam delineados devem ter em conta esta discrepância que dificulta a avaliação do impacto da violência entre irmãos e contribui para o desconhecimento do fenómeno (Straus, 2007).

Finalmente, algumas limitações do presente estudo devem ser tidas em consideração. Por se tratar de um estudo transversal retrospectivo impede, desde logo, extrapolações de causalidade e pode introduzir um viés que não deve ser ignorado, uma vez que se solicita um recuo temporal relativamente importante. $\mathrm{O}$ recrutamento de uma amostra unicamente universitária, dada a sua homogeneidade, não permite também a generalização de resultados. O facto 
de os participantes serem instruídos a classificar as suas respostas de acordo com os cinco termos (isto é, "conflito", "rivalidade", "agressão", "violência" e "abuso") condiciona naturalmente as suas respostas (Kettrey \& Emery, 2006), pelo que futuros estudos deverão ter este aspecto em consideração. A título de exemplo, os participantes podem ser convidados a rotularem as experiências que têm com os seus irmãos (Kettrey \& Emery, 2006), sem lhes ser dado qualquer instrução. Uma outra limitação prende-se com a desejabilidade social. Os participantes podem ter respondido de acordo com o que é desejável socialmente, considerando os comportamentos como "rivalidade" (Hardy et al., 2010) e não de acordo com o que efetivamente aconteceu. Nesse sentido, em posteriores trabalhos, recomenda-se o estudo e controlo da desejabilidade social que possa estar associada às respostas dadas.

\section{Conclusão}

Em suma, este estudo alerta para a elevada prevalência de violência entre irmãos, numa grande amostra universitária portuguesa. Particularmente, é crucial que se considere o facto das próprias vítimas atribuírem os abusos à "rivalidade fraterna", contribuindo assim, inadvertidamente e de forma claramente dissonante, para o desconhecimento dos limites da violência entre irmãos e, indiretamente, na família. Deste modo, parece-nos que seria importante o desenvolvimento de campanhas de sensibilização sobre o fenómeno, à semelhança do que tem sido feito com outras formas de violência doméstica (conjugal e parental), com o intuito de alertar para a sua elevada ocorrência. Sendo assim, os profissionais estariam mais predispostos para detectar/ sinalizar eventuais situações de risco. Uma outra implicação prende-se com o facto de a rivalidade ser utilizada para explicar comportamentos abusivos entre os irmãos, pelo que esta justificação poderá ser um sintoma de alerta para profissionais (de diversas áreas) sempre que surja nas narrativas de adultos ou crianças/jovens. Será igualmente útil alargar a avaliação da percepção de violência aos outros subsistemas familiares (ex., conjugal, parental), de forma a percebermos se a dissonância cognitiva é específica do relacionamento entre os irmãos ou se também acontece nesses subsistemas. Finalmente, investigações futuras devem privilegiar estudos longitudinais que abranjam diferentes faixas etárias e comparem diferentes amostras (i.e., clínicas versus comunitárias) de modo a compreender a forma como as fratrias evoluem. Este fenómeno carece igualmente de estudos qualitativos, sobretudo centrados na análise dos significados atribuídos aos diversos conceitos utilizados na explicação do fenómeno: rivalidade, conflito, agressão, violência ou abuso.

\section{Referências}

Alpert, J. L. (1997). Sibling child sexual abuse research review and clinical implications. Journal of Aggression, Maltreatment \& Trauma, 1(1), 263-275. doi:10.1300/J146v01n01_14
Caffaro, J. V., \& Conn-Caffaro, A. (1998). Sibling abuse trauma: Assessment and intervention strategies for children, families, and adults. New York: Haworth Press.

Daie, N., Witztum, E., \& Eleff, M. (1989). Long-term effects of sibling incest. Journal of Clinical Psychiatry, 50, 428-431.

Dawson, J. M., \& Langan, P. A. (1994). Murder in families. Washington DC: Bureau of Justice Statistics.

Eriksen, S., \& Jensen, V. (2009). A push or a punch: Distinguishing the severity of sibling violence. Journal of Interpersonal Violence, 24, 183-208. doi:10.1177/0886260508316298

Fernandes, O. M. (2002). Semelhanças e diferenças entre irmãos. Lisboa: Climepsi Editores.

Finkelhor, D. (1980). Sex among siblings: A survey of prevalence, variety, and effects. Archives of Sexual Behavior, 9, 171-194. doi:10.1007/BF01542244

Finkelhor, D., Turner, H., \& Ormond, R. (2006). Kid's stuff: The nature and impact of peer and sibling violence on younger and older children. Child Abuse \& Neglect, 30, 1401-1421. doi:10.1016/j.chiabu.2006.06.006

Graham-Bermann, S., Cutler, S., Litzenberger, B., \& Schwartz, W. (1994). Perceived conflict and violence in childhood sibling relationships and later emotional adjustment. Journal of Family Psychology, 8, 85-97. doi:10.1037/0893-3200.8.2.224

Hamby, S. L., \& Grey-Little, B. (2000). Labeling partner violence: When do victims differentiate among acts? Violence \& Victims, 15(2), 173-186.

Hardy, M. S. (2001). Physical aggression and sexual behavior among siblings: A retrospective study. Journal of Family Violence, 3, 255-268. doi:10.1023/A:1011186215874

Hardy, M. S., Beers, B., Burgess, C., \& Taylor, A. (2010). Personal experience and perceived acceptability of sibling aggression. Journal of Family Violence, 25, 67-71. doi:10.1007/s10896009-9270-3

Hoffman, K. I., Kiecolt, K. J., \& Edwards, J. N. (2005). Physical violence between siblings: A theoretical and empirical analysis. Journal of Family Issues, 26(8), 1103-1130. doi:10.1177/0192513X05277809

Kettrey, H., \& Emery, E. (2006). The discourse of sibling abuse. Journal of Family Violence, 21, 407-416. doi:10.1007/s10896006-9036-0

Kiselica, M., \& Morrill-Richards, M. (2007). Sibling maltreatment: The forgotten abuse. Journal of Counseling \& Development, 85(2), 148-160. doi:10.1002/j.1556-6678.2007.tb00457.x

Kolko, D. J., Kadzin, A. E., \& Day, B. T. (1996). Children's perspectives in the assessment of family violence: Psychometric characteristics and comparison to parent's reports. Child Maltreatment, 1, 156-167. doi: 10.1177/1077559596001002007

Krienert, J. L., \&. Walsh, J. A. (2011a). My brother's keeper: A contemporary examination of reported sibling violence using national level data, 2000-2005. Journal of Family Violence, 26, 331-342. doi:10.1007/s10896-011-9367-3

Krienert, J. L., \&. Walsh, J. A. (2011b). Sibling sexual abuse: An empirical analysis of offender, victim, and event characteristics in National Incident-Based Reporting System (NIBRS) data, 2000-2007. Journal of Child Sexual Abuse, 20(4), 353-372. doi:10.1080/10538712.2011.588190

Noland, V. J., Liller, K. D., McDermott, R. J., Coutler, M. L., \& Seraphine, A. E. (2004). Is adolescent sibling violence a precursor to college dating violence? American Journal of Health Behavior, 28, 13-23. 
Nunnally, J., \& Bernstein, I. (1994). Psychometric theory (3rd ed.). New York: McGraw-Hill.

Phillips, D., Phillips, K. H., Grupp, K., \& Trigg, L. (2009). Sibling violence silenced: Rivalry, competition, wrestling, playing, roughhousing, benign. Advances in Nursing Science, 32(2), 1-16.

Relva, I. C., Fernandes, O. M., \& Alarcão, M. (2012). Violência entre irmãos: Uma realidade desconhecida. Interamerican Journal of Psychology, 46(3), 205-214.

Relva, I. C., Fernandes, O. M., Alarcão, M., \& Martins, A., (2014). Estudo exploratório sobre a violência entre irmãos em Portugal. Psicologia: Reflexão e Crítica, 27(2), 398-408. doi: 10.1590/ 1678-7513.201427551

Relva, I., Fernandes, O. M., \& Costa, R. (2013). Psychometric Properties of Revised Conflict Tactics Scales: Portuguese Sibling Version (CTS2-SP). Journal of Family Violence, 28(6), 577-585. doi: 10.1007/s10896-013-9530-08

Smith, H., \& Israel, E. (1987). Sibling incest: A study of the dynamics of 25 cases. Child Abuse \& Neglect, 11, 101-108. doi:10.1016/0145-2134(87)90038-X

Stock, L. (1993). Sibling abuse: It's much more serious than child's play. Children's Legal Rights Journal, 14, 19-21.
Straus, M. A. (2007). Conflict Tactics Scales. In N. A. Jackson (Ed.), Encyclopedia of domestic violence (pp. 190-197). New York: Routledge, Taylor \& Francis Group.

Straus, M. A., Gelles, R. J., \& Steinmetz, S. K. (1980). Behind closed doors. Violence in the American family. Garden City: Anchor Books.

Straus, M. A., Hamby, S. L., Boney-McCoy, S., \& Sugarman, D. B. (1996). The Revised Conflict Tactics Scales (CTS2): Development and preliminary psychometric data. Journal of Family Issues, 17, 283-316. doi:10.1177/019251396017003001

Toman, W. (1993). Family constellation: Its effects on personality and social behavior. New York: Springer Publishing.

Turner, H. A., Finkelhor, D., Ormrod, R., \& Hamby, S. L. (2010). Infant victimization in a nationally representative sample. Pediatrics, 126(1), 44-52. doi:10.1542/peds.2009-2526

Underwood, R. C., \& Patch, P. C. (1999). Siblicide: A descriptive analysis of sibling homicide. Homicide Studies, 3(4), 333-348. doi:10.1177/1088767999003004005

Wiehe, V. R. (1997). Sibling abuse: Hidden physical, emotional and sexual trauma (2nd ed.). Thousand Oaks, CA: Sage Publications.

Wiehe, V. R. (2002). What parents need to know about sibling abuse. Utah: Bonneville Books.

Recebido em 24.01.2013

Primeira decisão editorial em 28.11.2013

Versão final em 18.12.2013

Aceito em 19.02.2014 